\title{
SUR CERTAINES PARTICULARITÉS BIOLOGIQUES D'UN TRYPANOSOME DE LA GRENOUILLE VERTE TRYPANOSOMA INOPINATUM SERGENT, 1904
}

\author{
Par Alice BUTTNER et Nicole BOURCART
}

Découvert par Edmond et Etienne Sergent, en 1904, dans le sang d'une Rana esculenta capturée en Kabylie, le Trypanosoma inopinatum fut ensuite observé à Constantine, la même année, par A. Billet.

C. França et M. Athias, en 1906, ont retrouvé ce Trypanosome au Portugal; cette souche géographique présente une virulence atténuée par rapport à la souche algérienne qui, expérimentalement, détermine chez les grenouilles vertes des infections aiguës, le plus souvent mortelles, alors que la souche portugaise ne donne en général que des affections chroniques.

W. S. Patton, aux Indes, signalait à son tour (1908), chez Rana tigrina, un Trypanosome sanguicole auquel il donnait le nom de Trypanosoma hendersoni, espèce qui a été rapportée ultérieurement par Brumpt et França $(1909,1911,1915)$ à l'inopinatum.

Le cycle évolutif de ce Flagellé a été élucidé, en 1906, par E. Brumpt. Billet avait déjà montré que ce parasite vivait dans le tube digestif d'une sangsue Rhynchobdelle, Helobdella algira, qui se nourrit du sang de Batraciens Anoures. E. Brumpt décrivit les modalités de ce développement chez ses deux hôtes successifs, $H$. algira et $R$. esculenta ; en 1907, il démontra que cette infection pouvait être héréditaire chez les sangsues parasitées. De son côté, C. França $(1912,1915)$ a suivi l'évolution du Trypanosome portugais chez l'hôte vertébré, mais les conclusions auxquelles il aboutit sont notablement différentes de celles publiées par E. Brumpt pour la souche algérienne.

Possédant depuis 1948 une souche algérienne de $T$. inopinatum (1), nous nous sommes proposé d'étudier à nouveau le comportement de ce Flagellé et d'examiner dans quelle mesure les

(1) Souche qui nous a été aimablement envoyée par le Professeur Ed. Sergent.

Ann. de Parasitologie, T. XXX, $\mathrm{N}^{\circ} 5-6 .-1955$. 
observations d'E. Brumpt et de C. França sont éventuellement conciliables. Par ailleurs, il nous est apparu que l'évolution de be Trypanosome présentait des modalités curieuses et qu'une différen ${ }^{\circ *}$ ciation s'opérait chez les formes évolutives au cours de la 'č. sance, donnant à celles-ci des significations physiologiques et epidémiologiques précises que nous nous efforcerons d'analyser ici.

\section{Matériel et techniques}

L'étude des formes évolutives chez l'hôte vertébré a étć faite sur des R. esculenta var. ridibunda originaires de Vendée, indemnes de cette trypanosomose qui n'existe pas en France. L'infestation des Rana a été réalisée, soit par inoculation d'1/2 cc. de dilution de sang infectieux dans du citrate de soude, à raison de trois à cinq Trypanosomes par champ, soit par piqûre de sangsues parasitées. Les expériences ont été poursuivies à une température variant entre 20 et $25^{\circ} \mathrm{C}$. Des coupes histologiques d'organes et des frottis par apposition, áinsi que des frottis de sang, lymphe, moelle osseuse, ont été eflectués périodiquement du $5^{\mathrm{e}}$ au $35^{\mathrm{e}}$ jour de l'infestation.

L'évolution chez la sangsue a été suivie chez une trentaine d'H. algira neuves d'élevage (1), depuis la $4^{e}$ heure après l'ingestion de sáng parasité jusqu'au $15^{\circ}$ jour, à des températures variables $\left(8\right.$ à $25^{\circ} \mathrm{C}$.). Des frottis du contenu stomacal et de la gaine de la trompe ont été faits après 4 , $6,9,14,17,31$ et 62 heures, 3 jours $1 / 2,5$ jours et 15 jours. Coloration des frottis de sang ou de sangsue au May-Grünwald-Giemsa, et des organes de Rana à l'hémalun-éosine, trichrome au ponceau de xylidine-bleu d'aniline, et méthode de Mallory.

\section{Etude du développement}

Rappelons rapidement les données actuellement acquises sur le cycle évolutif de ce Trypanosome :

Chez la grenouille, les Trypanosomes inoculés par la sangsue se multiplient d'abord au point d'inoculation dans le tissu conjonctif, puis ils apparaissent dans le sang au bout de quelques jours (petites formes), grandissent (formes intermédiaires $=T$. elegans de França), pour aboutir à des formes géantes à court flagelle $(=T$. undulans de França), qui se localisent surtout dans les viscères (ventricule cardiaque en particulier) et se montrent peu dans la circulation périphérique. Ils se divisent par scissiparité. França (1915) fait état dans des frottis de rate et de poumon d'éléments leishmaniens, tantôt libres, tantôt inclus dans des mononucléaires, parfois en voie de division. Il signale également une

(1) Technique d'isolement d'une souche neuve d' $H$. algira (voir $\mathrm{N}$. Bourcart, ces Ann., N. et I., p. 504). 
8 noyaux et 4 centrosomes. França interprète ces éléments

7) comme devant constituer \& la transition entre les crithidies e invertébré et la première génération de Trypanosomes de ertébré ». Il mentionne en outre la présence épisodique, dans les ons anciennes, de grandes formes aflagellées à plusieurs noyaux. tes ces formes de division multiple n'ont jamais été reconnues par

Brumpt.

Chez la sangsue, d'après E. Brumpt, les Trypanosomes ingérés se cantonnent exclusivement dans les cæcums stomacaux, où ils se transforment en Crithidia, parfois en Leptomonas, pour remonter ensuite dans la trompe, où ils s'accumulent sous la forme Trypanosoma (Trypanosomes métacycliques', seules formes infestantes pour le vertébré.

Reprenant cette évolution, nous donnons ci-dessous le détail de nos observations :

\section{1) Ghez Rana esculenta var. ridibunda}

a) Evolution dans le sang périphérique. - Les parasites inoculés, soit à partir de sangsues parasitées, soit par dilution de sang infectieux, n'apparaissent dans le sang que vers le $4^{\circ}$ ou $5^{\circ}$ jour, quand la température est voisine de $25-28^{\circ} \mathrm{C}$.

Ces jeunes formes sanguicoles (fig. 1, $a, b, c$ ), très mobiles, ont leur membrane ondulante plus développée et sont moins effilées que les Trypanosomes métacycliques de la sangsue (fig. 5, $x, y, z$ ). Elles se divisent activement par scissiparité. Vers le $10^{\circ}$ jour, la température restant constante, des formes intermédiaires s'observent dans le sang ; la division est toujours binaire, mais moins active, et des Trypanosomes géants, au flagelle épais et court, deviennent visibles à partir du $15^{\circ}$ jour à côté des éléments plus jeunes (fig. $1, d$ ). Ces grands Trypanosomes, rares dans le sang périphérique, se réfugient dans le sang profond. Au terme du développement, ils sont presqu'immobiles et ne se divisent plus : ce sont les formes « adultes »(1), pour reprendre l'expression attribuée à M. Robertson par G. Lavier et qui, d'après ce dernier auteur, « n'implique aucune aptitude spéciale à la division, ni chez le vertébré, ni chez l'invertébré ». Ces formes adultes représentent en fait les stades terminaux de l'évolution chez l'hôte vertébré.

b) Evolution dans les organes. - Au bout de quelques jours ( 7 à 15 jours suivant la température), les frottis et coupes d'organes font apparaître dans les mononucléaires et les cellules histiocy-

(1) Terminologie que nous n'adoptons qu'à regret puisqu'il n'y a pas ici de processus sexué. 
taires des foyers parasitaires où les Flagellés se retrouvent forme Leishmania ; la rate est particulièrement intéressée degrés variables, le foie, les poumons, les reins. Ces Lei: semblent se diviser par une pseudo-schizogonie, car on pet vidualiser des éléments à 3 et 4 noyaux et blépharoplastes, s bres, soit intracellulaires (fig. 2, $a, d$ ). L'aspect histologique de
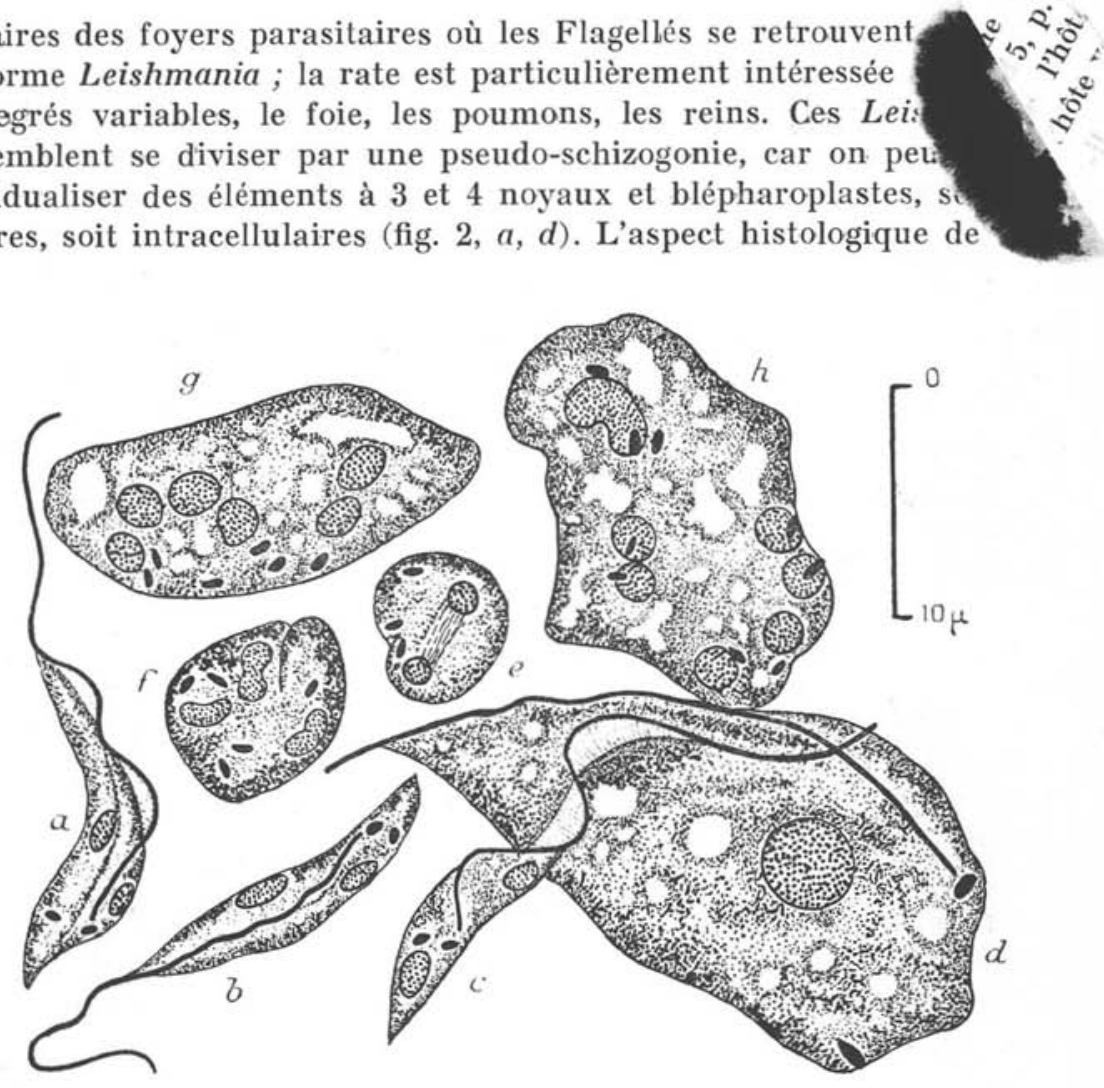

Fig. 1. - Formes de division chez Rana esculenta : $a, b$, $c$, petites formes ; $d$, adulte ; $e, f, g, h$, grandes formes aflagellées plurinucléées de la rate.

formes de multiplication (cytoplasme très basophile, noyaux condensés, blépharoplastes allongés et bien délimités) les différencie nettement des formes de phagocytose, souvent très nombreuses, où les parasites dégénérés sont partiellement digérés dans des vacuoles cytoplasmiques (fig. $2, c$ ).

Les stades de transition qui succèdent à la colonisation cellulaire, lors du retour à la forme Trypanosome, s'observent en général entre le $10^{\circ}$ et $20^{\circ}$ jour, suivant l'élévation de la température : des amas leishmaniö̈des apparaissent au voisinage des mononucléaires désertés, la plupart éclatés, et certains éléments présentent parfois déjà un court flagelle (fig. 2, d) ; ils deviennent bientôt Crithidia, 


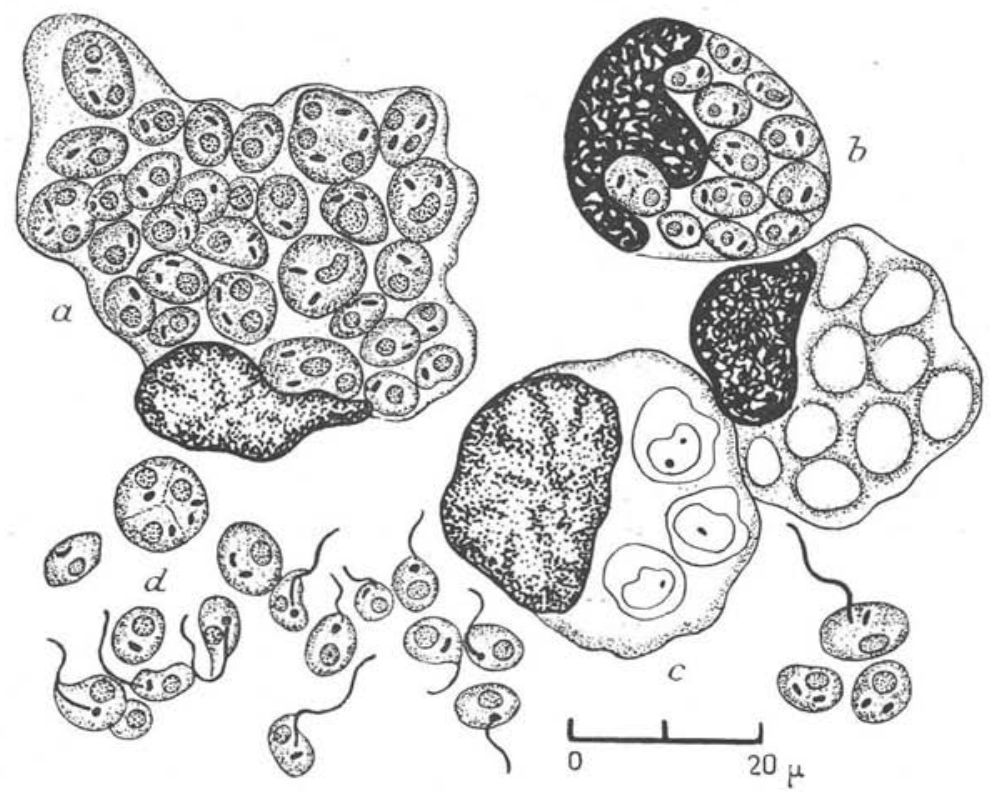

Fig. 2. - Evolution chez R. esculenta : $a, b$, division intracellulaire dans les mononucléaires de la rate ; $c$, phagocytose ; $d$, passages de la forme Leishmania à la forme Trypanosome.

puis ces Flagellés piriformes acquièrent progressivement la gracilité des Trypanosomes sanguicoles. La séquence de ces transformations est tout à fait semblable à celle observée pour T. cruzi (fig. 3).

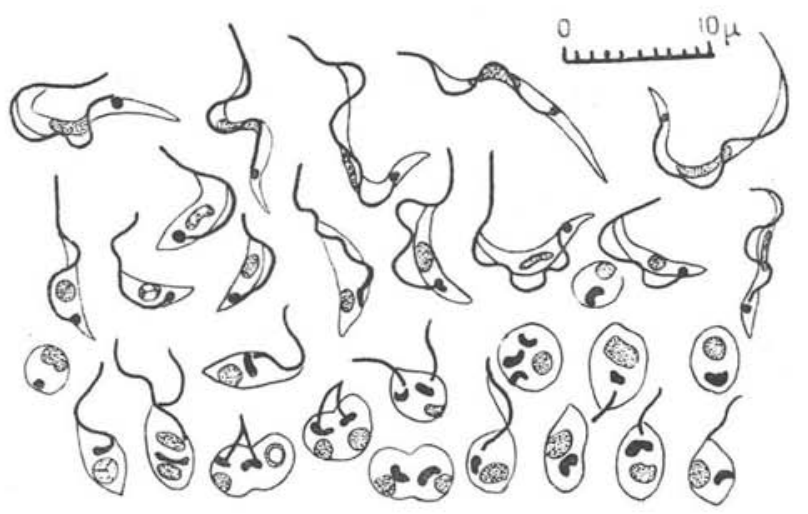

Frg. 3. - T , cruzi : Frottis de muscle cardiaque d'un rat: passages de la forme Leishmania à la forme Trypanosome (d'après E. Brumpt). 
Lorsque l'infestation est intense, la rate est en partie détruite; mais, dans les manifestations chroniques, la rate régénère et conserve pendant plusieurs semaines quelques foyers de Leishmania.

Dans les infestations anciennes (2 à 3 mois), de grandes formes sans flagelles, à $4,5,6$ ou 8 noyaux et blépharoplastes (fig. $1, e, f$, $g, h$ ), libres ou intracellulaires, envahissent la rate, parfois d'autres organes (foie, cœur), et semblent y persister longtemps.

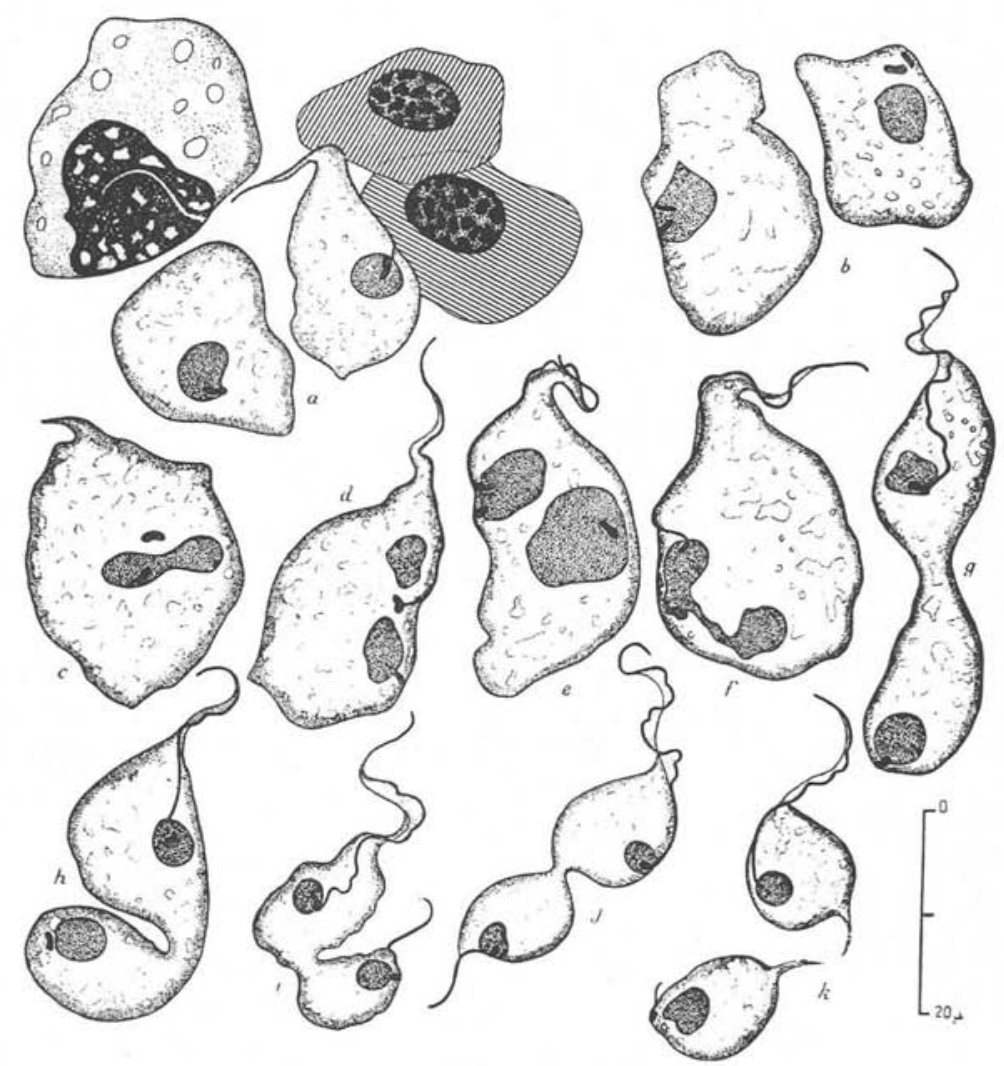

Fig. 4. - Evolution chez la sangsue :

$a \ldots k$, premières divisions des formes adultes

\section{2) Ghez Helobdella algira}

L'infestation d' $H$. algira neuves sur des $R$. ridibunda, présentant dans leur sang des Trypanosomes à divers stades de développement, nous a permis de constater que, seules, les grandes formes adultes évoluaient chez ces Hirudinées. Les petites formes sangui- 


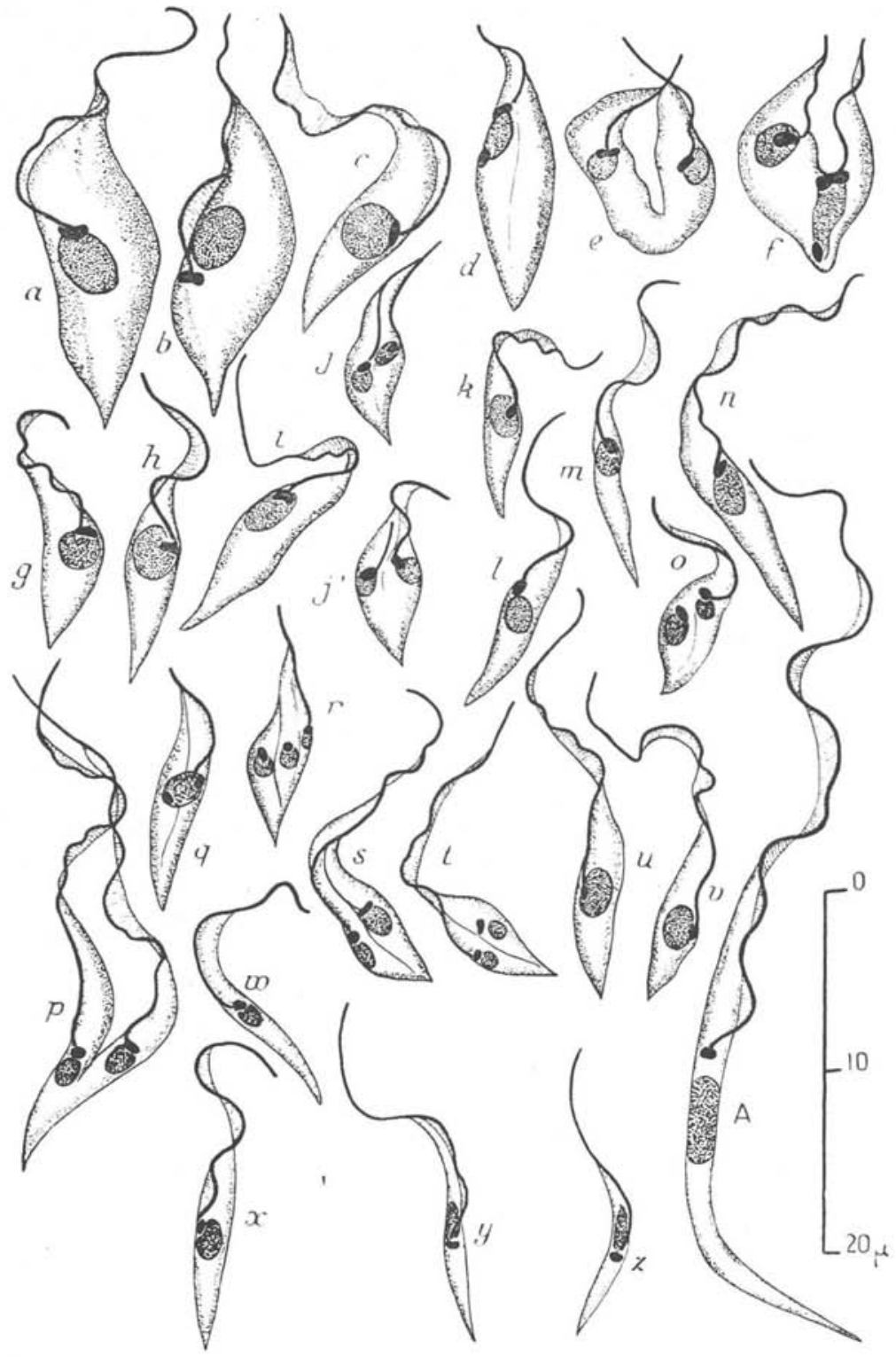

Fig. 5. - Fin de l'évolution cyclique chez la sangsue : $a, b$, formes en fuseau : $c \ldots . w$, divisions ultérieures sous la forme $\mathrm{Cri}$ thidia ; $w, x$, formes Crithidia persistant longuement dans la gaine de la trompe; $y, z$, Trypanosomes métacycliques; A, une forme allongée, crithidienne, de résistance, 
coles dégénèrent et disparaissent en 24 à 72 heures. Les Trypanosomes adultes commencent à se diviser 4 heures après l'ingestion du sang parasité ; cette division est binaire ; elle débute par celle du blépharoplaste, se poursuit par celle du noyau et s'achève par une cytodiérèse produite par un étranglement médian du cytoplasme (fig. 4). Des formes Crithidia apparaissent dès la première division, parfois chez l'une seulement des formes qui en sont issues, l'autre demeurant encore Trypanosome (fig. 4, g). Les divisions ultérieures aboutissent à des formes en fuseau moins volumineuses, quelques-unes encore Trypanosomes (fig. 5, b), les autres Crithidia (fig. 5, a). Le processus se poursuit, donnant des formes de plus en plus grêles, qui sont toutes Crithidia (fig. 5, $c$ à $x$ ). A la phase ultime, elles remontent et viennent s'accumuler dans la gaine de la trompe, ainsi qu'E. Brumpt l'a signalé. Le nombre des parasites s'est alors sensiblement accru. Ils demeurent souvent longtemps sous cette forme avant de se transformer en Trypanosomes métacycliques, et peuvent vraisemblablement être inoculés à ce stade à l'hôte vertébré sans que leur développement ultérieur en soit influencé. En effet, ces formes Crithidia, dont le blépharoplaste, en voie de migration, est généralement accolé au bord antérieur du noyau (fig. $5, u, x$ ), diffèrent très peu du Trypanosome métacyclique dont le blépharoplaste est encore tout proche de la base du noyau (fig. $5, y, z$ ).

Quand la température s'abaisse au-dessous de $10^{\circ}$, au cours de l'évolution cyclique chez la sangsue, on voit apparaitre dans les cæcums stomacaux des formes allongées crithidiennes, de très grande taille (fig. 5, A), qui semblent l'homologue des formes de résistance Leptomonas, signalées dans les mêmes conditions par E. Brumpt.

\section{Discussion et conclusion}

L'étude détaillée de ce développement suggère certaines réflexions relatives à diverses particularités biologiques de ce Trypanosome :

1) Tout d'abord, l'intervention à tous les stades évolutifs de modifications, qui les différencient morphologiquement et physiologiquement, fait qu'à aucun moment les formes qui se succèdent ne sont interchangeables. Chacune d'elles est étroitement liée à une fraction du cycle, et l'ensemble s'articule comme un véritable processus de maturation dont l'aboutissement serait le Trypanosome adulte. Ce dernier, comme l'observe G. Lavier (1942-1943), réunit le maximum de détails structuraux et " réalise en somme l'épanouissement morphologique de l'espèce $\gg$. 
Cette adaptation étroite et cette différenciation des stades sont faciles à mettre en évidence : c'est ainsi qu'après multiplication chez la sangsue sous la forme Crithidia, les Trypanosomes métacycliques qui en dérivent ne peuvent plus se reproduire chez cet invertébré ; mais, inoculés par lui à un hôte spécifique, la grenouille verte, ils voient renaître chez elle leurs potentialités évolutives et se transforment aussitôt en Trypanosomes sanguicoles qui se divisent activement. Ces jeunes formes sanguicoles sont différentes des formes métacycliques; elles ne peuvent plus vivre chez la sangsue et sont déjà adaptées à l'hôte vertébré, ce qui se manifeste par une spécificité moins stricte, leur permettant d'évoluer chez une espèce voisine, $R$. temporaria. Celle-ci, en effet, sensible à l'inoculation de sang infectieux, ne s'infeste pas par piqûre de sangsue parasitée, c'est-à-dire par inoculation de Trypanosomes métacycliques.

Les formes intermédiaires montrent à leur tour des potentialités modifiées : elles croissent progressivement et leur pouvoir de division s'affaiblit parallèlement. Lorsqu'elles deviennent adultes, elles perdent leur pouvoir de division qui reparaîtra seulement après passage chez l'hôte invertébré et sera le point de départ de nouvelles générations.

Bien qu'il soit souvent hasardeux de généraliser, on peut se demander si cette spécialisation des stades évolutifs n'est pas un phénomène constant chez tous les Trypanosomes qui présentent une durée de développement suffisante pour atteindre le stade adulte chez l'hôte vertébré. Tel serait le cas des Trypanosomes de Poissons, d'Amphibiens, de Reptiles, peut-être de certains Oiseaux ou même de Mammifères. Il semble, en revanche, qu'un raccourcissement de la vie individuelle, tel qu'il se manifeste chez certaines espèces pathogènes de Mammifères, entraine ipso facto une perte partielle de la spécialisation et une certaine polyvalence des formes sanguicoles ; celles-ci ont acquis un pouvoir quasi-illimité de division et semblent avoir perdu la capacité de se transformer en adultes, mais elles sont susceptibles d'évoluer aussi bien chez l'hôte vertébré que chez l'hôte vecteur (T. brucei, $T$. gambiense, $T$. rhodesiense, T. cruzi).

2) A côté d'une reproduction libre par division binaire dans le sang de la grenouille, le $T$. inopinatum montre une aptitude curieuse à se multiplier par voie intracellulaire. Ce fait, nous l'avons relaté, avait déjà été observé par França en 1912 ; mais la figure donnée par cet auteur (1915) s'apparente visiblement à la phagocytose, et le phénomène a toujours été interprété comme tel par E. Brumpt. Cependant, Wenyon (1926) fait état de ce mode de 
reproduction; comme França, il admet que ces éléments en division représentent la phase initiale du développement chez la grenouille, préludant à l'apparition dans le sang périphérique des formes juvéniles flagellées ; en outre, le processus de reproduction est assimilé par lui au mode de division binaire, intracellulaire, de T. cruzi : «It would thus appear that reproduction takes place by division of Leishmania forms in the lung or other organs in much the same way as occurs in T. cruzi. The Leishmania forms are apparently derived in the first place from the flagellates inoculated by the leech. »

A notre avis, ce mode de reproduction ne parait pas s'inscrire précocement dans le cycle, car il ne s'observe que lorsque les formes juvéniles sont présentes dans le sang depuis déjà plusieurs jours. Il ne peut pas non plus être confondu avec la phagocytose, souvent intense, en raison de la basophilie des éléments intracellulaires, de leur activité de division (fig. 2, $a$ et $b$ ), et de tous les stades intermédiaires observés lors du retour à la forme Trypanosome (fig. 2, $d$ ). D'autre part, les formes intra- ou extracellulaires, à $2,3,4$ noyaux et même davantage, témoignent d'un processus divisionnel beaucoup plus proche de la schizogonie que de la division binaire des Leishmania de T. cruzi.

Rappelons à cet égard le cas de T. parroti, parasite du Discoglossus pictus: la reproduction s'opère à l'intérieur des cellules hépatiques par division multiple (somatelle à courts flagelles) (E. Brumpt, 1936 ; P.-P. Grassé, 1953). Le processus n'est done pas nouveau pour un Trypanosome de Batracien, mais, lorsqu'il existe, c'est à l'exclusion de toute autre division dans le sang. L'originalité de $T$. inopinatum serait de présenter à la fois les deux modes de division chez l'hôte vertébré.

Quelle pourrait-être la signification de cette division intracellulaire, apparemment multiple ? Constitue-t-elle un mode subsidiaire de division ou a-t-elle dans le cycle une fonction particulière ?

Observons qu'elle intervient à un moment précis, celui qui sépare l'apparition des premiers Trypanosomes de celle des formes moyennes dans le sang. Nous inclinons à penser qu'il s'agit de divisions de maturation intervenant entre les stades juvéniles et les formes moyennes; en effet, on ne trouve pas d'individus intermédiaires entre petits et moyens Trypanosomes, alors qu'on observe au contraire des tailles très variées, aussi bien parmi les Leishmania que chez leurs intermédiaires de passage à la forme Trypanosome. La division binaire apparait plutôt comme un moyen de multiplication rapide d'individus au même stade. 
Lorsque le stade des formes moyennes est atteint, la croissance semble s'effectuer à partir du Trypanosome lui-même et de façon très progressive, car on retrouve alors toutes les tailles intermédiaires entre les Trypanosomes moyens et les grandes formes adultes.

Quant aux grandes formes polynucléées d'apparition tardive, leur rôle n'apparaît pas nettement, car elles sont inconstantes. Peut-être constituent-elles des foyers de résistance latents, qui seraient à l'origine des rechutes observées lors de déficiences organiques, après stabilisation de l'infection, peut-être s'agit-il simplement de formes de dégénérescence.

Cette double modalité de reproduction d'un Trypanosome sanguicole présente encore un intérêt systématique dans la discussion sur les coupures génériques proposées par différents auteurs pour le genre Trypanosoma. C'est ainsi que le genre Schizotrypanum, fréquemment adopté pour les Trypanosomes qui se multiplient chez le vertébré par voie intracellulaire (E. Dias, 1934), par opposition à ceux qui se reproduisent librement par division binaire, ne se justifie plus s'il existe des espèces présentant à la fois les deux types de reproduction chez leur hôte vertébré.

3) Existe-t-il une relation entre les formes évolutives du $T$. inopinatum et la pathogénicité très variable de ce Flagellé pour son hôte définitif ?

Nos observations viennent ici confirmer en grande partie les opinions générales émises par G. Lavier (1942-1943), lors de son étude sur les «Trypanosomes pathogènes de Mammifères »: avec la pathogénicité apparaissent deux caractères biologiques concomitants, le pouvoir indéfini de division des formes juvéniles et un certain fléchissement de la spécificité.

Dans la trypanosomose à inopinatum, la forme aiguë, presque toujours mortelle, est liée à la multiplication intense et continue des jeunes formes sanguicoles; par ailleurs, comme nous l'avons signalé, ces formes sanguicoles déterminent des infections mortelles chez la grenouille rousse, insensible aux formes métacycliques inoculées par la sangsue (baisse de la spécificité). L'évolution se poursuit néanmoins vers les formes adultes et, dans les infections expérimentales aiguës, on les retrouve en quantité considérable, agglutinées dans les organes, sous forme d'un liquide chyleux facilement observable par la ponction du cœur (1).

(1) L'anémie intense signalée toujours comme un signe pathognomonique de la maladie, n'est pas une conséquence directe de la pathogénicité des Trypanosomes. Elle est due à la présence des sangsues hématophages, dont les prélèvements sanguins ne sont pas compensés par un régime alimentaire réparateur.

AnN. de Parasitologie, T. XXX, $\mathrm{N}^{\circ} 5-6 .-1955$.

28. 
La prédominance des grandes formes caractérise au contraire la maladie chronique. Dans une étude antérieure (H. Galliard, A. Buttner et N. Bourcart, 1954), nous avons montré le rôle de la nutrition de l'hôte sur le développement du $T$. inopinatum, et obtenu expérimentalement chez des animaux non carencés, régulièrement nourris, le passage de la forme aiguë à la forme chronique, passage constaté par une diminution progressive des formes juvéniles. La résistance habituelle des grenouilles vertes d'Algérie à leurs infestations naturelles s'acquiert ainsi vraisemblablement grâce à une alimentation conforme à leurs besoins. Dans les infections expérimentales, les $R$. esculenta de France, maintenues en état de jeûne, ne peuvent acquérir cette prémunition et succombent plus ou moins rapidement.

Ceci semblerait prouver que, dans des conditions naturelles, ce Trypanosome n'est pas très pathogène. Spontanément, l'évolution se fait vers les formes adultes, c'est-à-dire vers la perte du pouvoir de multiplication, et la souche s'éloigne ainsi de la pathogénicité. Avec l'exaltation soudaine du pouvoir de reproduction des petites formes sanguicoles, due à un fléchissement de l'équilibre défensif de l'hôte, la souche tend au contraire vers une pathogénicité de plus en plus marquée. Un tel comportement rappelle à de nombreux égards celui de $T$. lewisi, parasite des rats, dont le pouvoir pathogène subit des variations qui semblent en rapport avec les mêmes facteurs.

\section{RÉSUMÉ}

1. T. inopinatum a été découvert par Ed. et Et. Sergent, en 1904, chez une grenouille verte, originaire de Kabylie.

Ce Trypanosome a été retrouvé par A. Billet à Constantine (1904), par C. França et M. Athias au Portugal (1906). Ces diverses souches géographiques, de virulence variable, ont été identifiées par E. Brumpt (1906) et C. França $(1909,1911,1915)$ à l'espèce inopinatum Sergent, 1904.

2. Le cycle évolutif de ce Trypanosome a été élucidé par E. Brumpt en 1906. Il requiert deux hôtes, un Hirudinée vecteur, Helobdella algira, et un hôte vertébré, Rana esculenta.

Les modalités curieuses du développement observées par nous chez une souche algérienne d'inopinatum, et les interprétations différentes de certaines parties du cycle données par E. Brumpt et C. França nous ont incitées à reprendre l'étude de cette évolution. 
3. L'étude du cycle chez la sangsue nous a permis de préciser que seuls les grands Trypanosomes évoluaient chez cet hôte. Des formes de résistance au froid, qui sont crithidiennes et non Leptomonas, ont été observées dans l'estomac. La longue persistance des Crithidia dans la gaine de la trompe (2 à 3 mois) permet de penser qu'ils sont parfois inoculés sous cette forme à l'hôte vertébré, et que le pouvoir infectieux apparait peut-être avant la transformation définitive en formes métacycliques.

4. L'évolution chez la grenouille emprunte deux types de multiplication :

a) division binaire libre, dans le sang, sous la forme Trypanosome ;

b) division multiple intracellulaire, dans les organes, sous la forme Leishmania.

La première est interprétée comme un mode de multiplication d'individus au même stade; la seconde comme une division de maturation, lors du passage des jeunes formes sanguicoles aux formes moyennes.

5. Diverses particularités biologiques nous ont frappées au cours de ce développement :

a) la différenciation des stades au cours de la croissance, donnant à chacun d'eux un rôle physiologique précis, strictement adapté à une fraction du cycle ;

b) le double mode de division du Trypanosome chez l'hôte vertébré, qui tendrait à rendre caduc le genre Schizotrypanum adopté par certains auteurs pour les Trypanosomes à multiplication intracellulaire chez le vertébré ;

c) la relation entre les formes évolutives et la pathogénicité de la souche: l'évolution normale vers les formes adultes et la perte du pouvoir de multiplication éloignent la souche de la pathogénicité ; l'exaltation soudaine de la division des jeunes formes sanguicoles marque au contraire une tendance plus ou moins accentuée vers la pathogénicité.

Les formes aiguës de la maladie sont en effet liées à la multiplication continue des formes juvéniles, alors que la prédominance des grandes formes adultes signale, à l'inverse, le caractère chronique de l'infestation. 


\section{BIBLIOGRAPHIE}

BH.LET (A.). - Sur le Trypanosoma inopinatum de la grenouille verte d'Algérie et sa relation possible avec les Drepanidium. C.R. Soc. Biol., LVI, 1904, vol. 2 , p. 161 .

Brumpt (E.). - Mode de transmission et évolution des Trypanosemes des poissons d'eau douce ; leur mode d'érolution. C.R. Soc. Bisl., XL, 1906, p. 162 .

- Expériences relatives au mode de transmission des Trypanosomes et des Trypanoplasmes par les Hirudinées. Ibid., XLI, 1906, p. 77.

- Rôle pathogène et mode de transmission du Trypanosoma inopinatum Ed. et Et. Sergent. Mode d'inoculation d'autres Trypanosomes. Ibid., XLI, 1906 , p. 167 .

- De l'hérédité des infections à Trypanosomes et à Trypanoplasmes chez les hôtes intermédiaires. Ibid., LXIII, 1907, p. 176.

Guérison de la maladie du sommeil chez le lérot vulgaire en hibernation. Action du froid sur le Trypanosoma inopinatum in vivo. Ibid., LXIV, 1908 , p. 1147.

Evolution de Trypanosoma lewisi, duttoni, nabiasi, blanchardi chez les puces et les punaises. Transmission par les déjections. Crmparaison avec $T$. cruzi. Immunité partielle dans les infections à Trypanosoma cruzi, transmission de ce Trypanosome par Cimex rotundatus. Ròle régulateur des hôtes intermédiaires. Passage à travers la peau. Bull. Soc. Path. Exot., VI, 1913, p. 167.

Un nouveau Trypanosome pathogène des vertébrés à sang froid, Trypanosoma parroti du Discoglossus pictus. C.R. Acad. Sci., CLXXXVI, 1928, p. 1160 .

Titres et travaux scientifiques, Paris, 1934, Masson et $\mathrm{C}^{\text {ie }}$ édit., p. 80 et suiv.

- Précis de Parasitologie, 5 édit., Paris, 1936, p. 300, et $6^{\circ}$ édit., Paris, 1949, Masson et $\mathrm{C}^{10}$ édit., p. 284 et suiv.

Dias (E.). - Trypanosoma cruzi ou Schizotrypanum cruzi? Mem. Inst. Osw. Cruz, XXIX, 1934, p. 203.

Revisão general dos hemoflagellados de Cheiropteros. Estudio experimental de Schizotrypanum de Phyllostomus hastatus : identidade com Schizotrypanum cruzi. O grupo vespertilionis. IX' Réunion Soc. argent. de Patol. reg. del Norte, Buenos-Aires, I, 1936, p. 10.

França (C.). - Le cycle évolutif des Trypanosomes de la grenouille (T. costatum, T. rotatorium et T. inopinatum). Arq. R. Inst. Bact. Camara Pestana, II, 1908, p. 89.

- Le cycle évolutif des Trypanosomes de la grenouille. Femarques à propos du travail de MM. W. S. Patton et C. Strickland. Ibid., II, 1909, p. 381.

- Sur la relation ontogénique entre les grands et les petits Trypanosomes de la grenouille. C.R. Soc. Biol., LXX, 1911, p. 978.

- Les formes aflagellées dans l'évolution d'un Trypanosome de Batracien (T. undulans). Bull. Soc. Path. Exot., V, 1912, p. 99.

- Le Trypanosoma inopinatum. Arch. f. Protist., XXXIV, 1915, p. 108. 
et Atrias (M.). - Notes cytologiques sur les Trypanosomes parasites de la grenouille (Rana esculenta). XV Congr. Internat. Med., sect. I, Anat., Lishonne, 1906, p. 394.

- - Recherches sur les Trypanosomes des Amphibiens. II. Le Trypanosoma rotatorinm de Hyla arborea. Arq. R. Inst. Bact. Camara Pestana, I, 1907 , p. 289.

Galliard (H.), Buttner (A.) et Bourcart (N.). - Action de la somatotrophine hypophysaire et de la nutrition sur l'évolution de Trypanosoma inopinatum Sergent, 1904 (souche algérienne) chez la grenouille verte. Ann. Parasit., XXIX, 1954, p. 179.

Grasse (P. P.). - Traité de Zoologie, tome I, fasc. 1, p. 631 et suiv., Paris, 1953, Masson et $\mathrm{C}^{\text {ie }}$ édit.

Laveran (A.) et Mesnil (F.). - Trypanosomes et trypanosomiases, Paris, 1904.

LAviER (G.). - L'évolution de la morphologie dans le genre Tryfanusoma. Ann. Paras., XIX, 1942-1943, p. 168.

Patton (W. S.). - Annual Report upon the work of bacteriological Section of the King Instilute of Preventive Medicine, Guindy, for the year 1907, Madras, 1908.

Posselle (A.). - La culture de Trypanosoma inopinatum, Trypanosome pathogène de la grenouille. Ann. Paras., I, 1923, p. 155.

Sergent (Ed. et Et.). - Sur un Trypanosome nouveau, parasite de la grenouille verte. C.R. Soc. Biol., LVI, 1904, p. 123.

Wenyon (C. M.). - Protozoology, Londres, 1926, p. 595 et suiv.

(Institut de Parasitologie de la Faculté de Médecine de Paris. Directeur: Prof. H. Galliard) 